\title{
Modeling of the Soluble Microbial Products (SMP) in Anaerobic Membrane BioReactors (AMBR): Equilibria and stability of the AM2b model *
}

\author{
B. Benyahia* T. Sari ${ }^{* *}$ J. Harmand ${ }^{* * *}$ B. Cherki ${ }^{* * * *}$ \\ * Laboratoire d'Automatique, Tlemcen University, Algeria and EPI \\ MERE, INRIA, Montpellier, France, (e-mail: benyahiab@yahoo.fr). \\ ** LMIA, Mulhouse University and EPI MERE, INRIA, Montpellier, \\ France, (e-mail: tewfik.sari@sophia.inria.fr) \\ *** LBE-INRA, UR050, Narbonne and EPI MERE, INRIA, \\ Montpellier, France, (e-mail: harmand@supagro.inra.fr). \\ **** Laboratoire d'Automatique, Tlemcen University, Algeria, (e-mail: \\ b_cherki@yahoo.fr)
}

\begin{abstract}
This paper deals with the modification of the AM2 model (Bernard et al. (2001)) to integrate the production and the degradation of the Soluble Microbial Products (SMP) in an Anaerobic Membrane BioReactor (AMBR). The developed model takes into account the characteristics of membrane bioreactors, namely i) there is no biomass in the effluent, ii) part of the SMP, corresponding to the biggest molecules, is retained in the reactional medium, iii) the decay rate and the withdraw of the biomass are considered. Possible equilibria of the system are calculated, and the influence of the maximum growth rate of the biomass on the SMP on these equilibria is analyzed. It is shown that the presence of this new variable in the model changes the qualitative properties of the classical AM2 model.
\end{abstract}

Keywords: Anaerobic digestion, Biotechnology, Equilibria, Fouling, Membrane BioReactor, SMP modeling.

\section{INTRODUCTION}

Effluent discharge constraints become more and more stringent and wastewater quantities to be treated increase. It is urgent to conceive high-performance treatment plants capable of rejecting in the natural environment good quality effluents possibly able to be reused in agriculture and/or industry. The technology of Anaerobic Membrane BioReactors (AMBR) appears promising to take up this challenge. However, the risk of membrane fouling limits the development of these systems. Modeling coupling the fouling phenomena together with the biotic characteristics, should allow to make these fouling more predictable and to act by means of a control in order to limit their impact on the system performance.

WWTP effluents contain a large variety of soluble organic compounds: residual substrates, intermediates or final products and Soluble Microbial Products (SMP) (Barker and Stuckey (1999)). The latter regroup organic compounds produced from the substrate metabolism (generally accompanied by a biomass growth) and from the biomass decay during the complete mineralization of simple substrates (Noguera et al. (1994)). Usually two categories of SMP are considered: BAP (Biomass Associated

* This work was supported by TREASURE (an INRIA Euromed $3+3$ project, http://www.treasure.fr), COADVISE (project FP7PEOPLE-IRSES-2008 No. 230833) and Aires-Sud (Tlemcen University and IRD) projects.
Products), associated with the biomass mortality, and UAP (Utilization Associated Products), associated with the substrate degradation and the biomass growth (Barker and Stuckey (1999)). The presence of SMP in a MBR affects the process performances (effluent quality) and they can pose problems of toxicity. In addition, their role in the membranes fouling is recognized. It is thus essential to integrate the SMP in the modeling of bioprocesses.

Most MBR are aerobic systems. This is why the efforts to couple biotic phenomena with membrane models have been primarily proposed for the aerobic systems. They are based on the modification of ASM models to include the SMP formation and degradation. On the contrary, in anaerobic conditions, only a few papers are available $(\mathrm{Ng}$ and Kim (2007)). Yet, anaerobic digestion systems have many advantages among which the possible use of the biogas produced as an energy source.

The first model to predict the production and the degradation of SMP in an anaerobic chemostat has been proposed by Noguera et al. (1994), in considering two categories of SMP, that are UAP and BAP. The model integrates the UAP production only starting from the initial organic matter (glucose) and considers their degradation only by the acidogenic bacteria. The BAP are considered to be produced by the decay of acidogenic bacteria and slowly degraded by these same bacteria. The model does not take into account neither the UAP and the BAP production starting from the acetate (intermediate product of the 
reaction), nor the decay of methanogenic biomass.

This model was further developed by Barker and Stuckey (2001) who have added i) the concept of the UAP production in consuming both the initial organic matter and the intermediate products by the acidogenic and methanogenic bacteria respectively, ii) the BAP production starting from the decay of acidogenic and methanogenic, and the iii) BAP degradation by acidogenic to grow and give intermediate products.

Latter on, Laspidou and Rittmann (2002) have proposed an unified theory for the production and the degradation of the UAP and the BAP, in considering the coupling between three compounds: Extracellular Polymeric Substances (EPS), SMP and inert biomass. They organize their theory through six hypotheses for how SMP, EPS, active and inert biomass are related.

Recently, Aquino and Stuckey (Aquino and Stuckey (2008)), have refined the model suggested in (Barker and Stuckey (2001)) by taking into account the concept of the EPS formation and degradation as suggested in Laspidou and Rittmann (2002), in order to predict the SMP production.

It should be stressed that the majority of the studies quoted above relate to conventional systems of anaerobic digestion (chemostat-like systems) and not to membrane reactors. In addition, the last developed models are rather complex and, clearly, are not adapted for control system design.

This paper aims at proposing i) a model for an AMBR with external membrane module, which should be simple enough to be used for control purposes and, in the same time, fine enough to allow linking in a pertinent way dynamics of fouling to SMP dynamics. To do so, we propose to modify the AM2 model (Bernard et al. (2001)) and to study equilibria of such a model including the SMP formation and degradation in an AMBR.

This paper is structured as follows. First, we present the model with SMP. Then, it is analyzed and its equilibria are characterized. Finally, simulation results are discussed before conclusions and perspectives are drawn.

\section{MATHEMATICAL MODEL}

\subsection{Hypotheses for the model development}

The figure 1 represents the compartments included in the model with $S_{1}$ the organic matter concentration (COD), $S_{1 \text { in }}$ the COD input concentration, $S_{2}$ the Volatile Fatty Acid concentration (VFA), $S_{2 i n}$ the VFA input concentration, $X_{1}$ the acidogenic biomass, $X_{2}$ the methanogenic biomass and $S=U A P+B A P$, the Soluble Microbial Products (SMP). The model is developed under the following assumptions (H1-H7). The figure 2 represents the matter separation by the membrane which is formalized through assumptions $\mathrm{H} 2, \mathrm{H} 3$ and $\mathrm{H} 6$.

Hypothesis 1. The reactional medium of the bioreactor is considered to be a homogeneous.

Hypothesis 2. The substrates $S_{1}$ and $S_{2}$ cross the external membrane without retention (the molecules size is smaller than the pore diameter).

Hypothesis 3. Total retention of biomasses $X_{1}$ and $X_{2}$ by the membrane is considered (the bacteria size is greater than the pore diameter). Therefore, there is no solids in

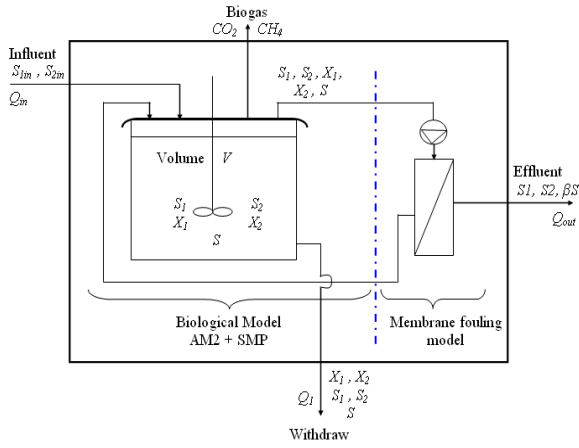

Fig. 1. Schematic representation of the proposed model

the effluent, and thus no term $-D X$ in the mass balance equation of $X_{i}$.

Hypothesis 4. Decay rates $D_{0}$ of biomasses are taken into account.

Hypothesis 5. The withdraw of the biomass with a flowrate $Q_{1}$ is considered.

Hypothesis 6. $\mathrm{SMP}=\mathrm{UAP}+\mathrm{BAP}$ are grouped into an unique variable of concentration $S$, which only a fraction $\beta$ leaves the bioreactor. The remaining, corresponding to macromolecules, is retained by the membrane. This is modeled in the mass balance equation by $-\beta D S$ where $0 \leq \beta \leq 1 \quad(\beta=0$ : total retention of SMP by the membrane; $\beta=1$ : free crossing of SMP through the membrane).

Hypothesis \%. The bioreactor is supposed to operate in normal conditions (no critical fouling: the flux is lower than the critical flux).

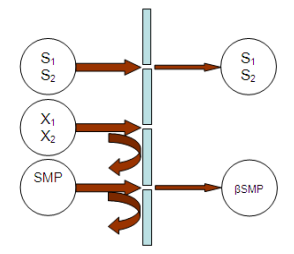

Fig. 2. Matter separation by the membrane

\subsection{Reaction schemes and mathematical equations}

Our model is based on the modification of the model AM2 that was developed within the framework of the European project $\mathrm{AMOCO}^{1}$ and discussed in Benyahia et al. (2010). The AM2 model considers only two functions of the anaerobic digestion (Bernard et al. (2001)).

i- Acidogenesis: the acidogenic bacteria $\left(X_{1}\right)$ consume the organic substrate $\left(S_{1}\right)$ and produce $\mathrm{CO}_{2}$ and VFA $\left(S_{2}\right)$; $k_{1} S_{1} \longrightarrow X_{1}+k_{2} S_{2}+k_{4} C_{2}$

ii- Methanogenesis: the methanogenic bacteria $\left(X_{2}\right)$ use $\mathrm{S}_{2}$ as substrate to grow, and produce $\mathrm{CO}_{2}$ and methane $\mathrm{CH}_{4}$.

$$
k_{3} S_{2} \longrightarrow X_{2}+k_{5} \mathrm{CO}_{2}+k_{6} \mathrm{CH}_{4}
$$

We propose to modify this reaction scheme in adding a new compound SMP named $S$. We model the SMP production

1 Acronym for Advanced MOnitoring and COntrol system for anaerobic processes, European FAIR project No. ERB-FAIR-CT96-1198 
from the degradation of $S_{1}, S_{2}$ and the decay of biomasses $X_{i}$. In addition, we consider the SMP degradation into $S_{2}$, $\mathrm{CH}_{4}$ and $\mathrm{CO}_{2}$ through the growth of $X_{1}$.

i- Acidogenesis + SMP Production: $k_{1} S_{1} \longrightarrow X_{1}+k_{2} S_{2}+b_{3} S+k_{4} C_{2}$ with reaction rate: $r_{1}=\mu_{1}\left(S_{1}\right) X_{1}$

ii- Methanogenesis + SMP Production: $k_{3} S_{2} \longrightarrow X_{2}+b_{4} S+k_{5} C_{2}+k_{6} \mathrm{CH}_{4}$ with reaction rate: $r_{2}=\mu_{2}\left(S_{2}\right) X_{2}$

iii- SMP Degradation: $b_{1} S \longrightarrow X_{1}+b_{2} S_{2}+k_{7} C_{2}$ with reaction rate: $r=\mu(S) X_{1}$

iv- SMP Production from biomass decay: $X_{1} \longrightarrow D_{0} S, \quad X_{2} \longrightarrow D_{0} S$

Let us note $\xi=\left[S_{1}, X_{1}, S_{2}, X_{2}, S\right]^{T}$ the state space vector of the model AM2+SMP (thereafter, we note this model AM2b). From this new reaction scheme and taking into account the above assumptions (H1-H7), the matter conservation principle enables us to write down the following mathematical model $\dot{\xi}=f(\xi)$.

$$
\begin{aligned}
\dot{S}_{1}= & D\left(S_{1 \text { in }}-S_{1}\right)-k_{1} \mu_{1}\left(S_{1}\right) X_{1} \\
\dot{X}_{1}= & {\left[\mu_{1}\left(S_{1}\right)+\mu(S)-D_{0}-D_{1}\right] X_{1} } \\
\dot{S}_{2}= & D\left(S_{2 i n}-S_{2}\right)-k_{3} \mu_{2}\left(S_{2}\right) X_{2}+\left[k_{2} \mu_{1}\left(S_{1}\right)+\right. \\
& \left.b_{2} \mu(S)\right] X_{1} \\
\dot{X}_{2}= & {\left[\mu_{2}\left(S_{2}\right)-D_{0}-D_{1}\right] X_{2} } \\
\dot{S}= & {\left[b_{3} \mu_{1}\left(S_{1}\right)+D_{0}-b_{1} \mu(S)\right] X_{1}+\left[b_{4} \mu_{2}\left(S_{2}\right)+\right.} \\
& \left.D_{0}\right] X_{2}-\left[\beta D+(1-\beta) D_{1}\right] S
\end{aligned}
$$

with:

$k_{1}$ : degradation rate of $S_{1}$ by $X_{1}[g / g]$

$k_{2}$ : production rate of $S_{2}$ by $X_{1}$ from $S_{1}[\mathrm{mmol} / \mathrm{g}]$

$k_{3}$ : degradation rate of $S_{2}$ by $X_{2}[\mathrm{mmol} / \mathrm{g}]$

$b_{1}$ : degradation rate of $S$ by $X_{1}[\mathrm{~g} / \mathrm{g}]$

$b_{2}$ : production rate of $S_{2}$ by $X_{1}$ from $S[\mathrm{mmol} / \mathrm{g}]$

$b_{3}$ : production rate of $S$ by $X_{1}$ from $S_{1}[\mathrm{~g} / \mathrm{g}]$

$b_{4}$ : production rate of $S$ by $X_{2}$ from $S_{2}[\mathrm{~g} / \mathrm{g}]$

$\beta$ : SMP fraction leaving the bioreactor

$D$ : dilution rate $\left(=\frac{Q_{i n}}{V}\right)\left[d^{-1}\right]$

$D_{0}$ : decay rate of biomass $\left[d^{-1}\right]$

$D_{1}$ : withdraw of biomass $\left(=\frac{Q_{1}}{V}\right)\left[d^{-1}\right]$

$S_{1 i n}$ : the COD input concentration $(g / l)$

$S_{2 i n}$ : the VFA input concentration $(\mathrm{mmol} / \mathrm{l})$

\section{MODEL EQUILIBRIA, STABILITY AND SIMULATION RESULTS}

\subsection{Model equilibria}

We complete the assumptions of the section 2.1 by the following hypothesis on the model kinetics.

Hypothesis 8 . The characteristics of the kinetics $\mu_{1}, \mu_{2}$ and $\mu$ are:

- $\mu_{1}(0)=\mu_{2}(0)=\mu(0)=0$ (no growth without substrate).

- $\mu_{1}^{\prime}\left(S_{1}\right)>0$ and $\mu^{\prime}(S)>0$ for $S_{1} \geq 0$ and $S \geq 0$ respectively.

- $\mu_{1}(\infty)=m_{1}$ and $\mu(\infty)=m$
- $\mu_{2}^{\prime}\left(S_{2}\right)>0$ if $0 \leq S_{2}<S_{2}^{M}$

- $\mu_{2}\left(S_{2}\right)$ reaches a maximum $\mu_{2}^{M}=\mu_{2}\left(S_{2}^{M}\right)$

- $\mu_{2}^{\prime}\left(S_{2}\right)<0$ for $S_{2}>S_{2}^{M}$

As examples of such functions, one considers the following Monod functions $\mu_{1}$ and $\mu$ and the Haldane function $\mu_{2}$.

$$
\begin{gathered}
\mu_{1}\left(S_{1}\right):=\frac{m_{1} S_{1}}{K_{1}+S_{1}}, \quad \mu(S):=\frac{m S}{K+S}, \\
\mu_{2}\left(S_{2}\right):=\frac{m_{2} S_{2}}{K_{2}+S_{2}+\frac{S_{2}^{2}}{K_{I}}} .
\end{gathered}
$$

To compute the equilibrium points, we cancel the derivatives (1) to (5). From (2) we have $X_{1}=0$ or $\mu_{1}\left(S_{1}\right)+$ $\mu(S)-D_{0}-D_{1}=0$, and from (4), we have $X_{2}=0$ or $\mu_{2}\left(S_{2}\right)-D_{0}-D_{1}=0$. Three cases must be considered.

- $X_{1}=0$. See Lemma 9 .

- $X_{1}>0$ and $X_{2}=0$. See Lemma 10 .

- $X_{1}>0$ and $X_{2}>0$. See Lemma 11.

Lemma 9. The equilibria $\left(S_{1}, X_{1}, S_{2}, X_{2}, S\right)$ of the system (1-5) for which $X_{1}=0$ are given by

- the washout equilibrium of $X_{1}$ and $X_{2}$, $E_{0}^{0}=\left(S_{1 i n}, 0, S_{2 i n}, 0,0\right)$, which always exists.

- the washout equilibrium of $X_{1}$ but not of $X_{2}$, $E_{0}^{i}=\left(S_{1 i n}, 0, \lambda_{2}^{i}, X_{2}^{i}, S^{i *}\right), i=1$ or 2 , where

$$
\begin{gathered}
X_{2}^{i}=\frac{D\left(S_{2 i n}-\lambda_{2}^{i}\right)}{k_{3}\left(D_{0}+D_{1}\right)}, \\
S^{i *}=\frac{b_{4}+\frac{D_{0}}{D_{0}+D_{1}}}{\left[\beta+(1-\beta) \frac{D_{1}}{D}\right] k_{3}}\left(S_{2 i n}-\lambda_{2}^{i}\right), \quad i=1,2
\end{gathered}
$$

Which exists if and only if $S_{2 i n}>\lambda_{2}^{i}$.

In the sequel we use the following notations. Let

$$
\begin{gathered}
F(S):=\mu_{1}^{-1}\left(D_{0}+D_{1}-\mu(S)\right) \\
G\left(S_{1}\right):=\frac{1}{B}\left[S_{1 i n}-S_{1}\right]\left[\frac{1}{k_{1}}\left(b_{3}+b_{1}\right)+\right. \\
\left.\frac{1}{k_{1} \mu_{1}}\left(D_{0}-b_{1}\left(D_{1}+D_{0}\right)\right)\right] \\
H_{i}\left(S_{1}\right):=\frac{1}{B}\left[A\left(S_{2 i n}-\lambda_{2}^{i}\right)+\left(S_{1 i n}-S_{1}\right)\left(\frac{A_{1}-A_{2}}{k_{1}}+\right.\right. \\
\left.\left.\frac{A_{2}\left(D_{1}+D_{0}\right)+D_{0}}{k_{1} \mu_{1}}\right)\right], \quad i=1,2
\end{gathered}
$$

Where $S_{2}=\lambda_{2}^{i}$ is a solution of equation $\mu_{2}\left(S_{2}\right)=D_{0}+D_{1}$ and,

$$
\left\{\begin{array}{l}
A=\frac{b_{4}\left(D_{0}+D_{1}\right)+D_{0}}{k_{3}\left(D_{0}+D_{1}\right)} \\
A_{1}=b_{3}+A k_{2}, A_{2}=A b_{2}-b_{1} \\
B=\beta+(1-\beta) \frac{D_{1}}{D}
\end{array}\right.
$$

Lemma 10. Let $\left(X_{1}, X_{2}, S_{1}, S_{2}, S\right)$ an equilibrium point of system (1-5). If $X_{1}>0$ and $X_{2}=0$ then one has $0<S_{1}<S_{1 i n}, S>0$ and $S_{2}>0$. Moreover $S_{1}$ and $S$ are solutions of equations system:

$$
\left\{\begin{array}{l}
S_{1}=F(S) \\
S=G\left(S_{1}\right)
\end{array}\right.
$$


and $X_{1}$ and $S_{2}$ are given by the formulas

$$
\begin{gathered}
X_{1}=D \frac{S_{1 i n}-S_{1}}{k_{1} \mu_{1}}, \\
S_{2}=S_{2 i n}+\left[k_{2} \mu_{1}+b_{2} \mu\right] \frac{S_{1 i n}-S_{1}}{k_{1} \mu_{1}} .
\end{gathered}
$$

Lemma 11. Let $\left(X_{1}, X_{2}, S_{1}, S_{2}, S\right)$ an equilibrium point of system (1-5). If $X_{1}>0$ and $X_{2}>0$ then one has $0<S_{1}<S_{1 i n}, S>0$ and $S_{2}=\lambda_{2}^{i}(i=1,2)$. Moreover $S_{1}$ and $S$ are solutions of equations system:

$$
\left\{\begin{array}{l}
S_{1}=F(S) \\
S=H_{i}\left(S_{1}\right), \quad i=1,2
\end{array}\right.
$$

and $X_{1}$ and $X_{2}$ are given by the formulas

$$
\begin{gathered}
X_{1}=D \frac{S_{1 i n}-S_{1}}{k_{1} \mu_{1}} . \\
X_{2}=D \frac{\left[S_{1 i n}-S_{1}\right]\left[k_{2} \mu_{1}+b_{2} \mu\right]+\left(S_{2 i n}-\lambda_{2}^{i}\right) k_{1} \mu_{1}}{k_{1} k_{3}\left(D_{0}+D_{1}\right) \mu_{1}} .
\end{gathered}
$$

With the following condition:

$$
\lambda_{2}^{i}<S_{2 i n}+\left(k_{2} \mu_{1}+b_{2} \mu\right) \frac{S_{1 i n}-S_{1}}{k_{1} \mu_{1}} .
$$

\subsection{Graphic calculation of equilibria}

The equilibrium points are obtained from the graphic calculation of the coordinates $\left(S_{1}, S\right)$ of the intersections of the graph of function $F$ with the graphs of functions $G$, $H_{1}$ and $H_{2}$.

The $F$ graph depends on the value of maximum growth rate $m$ of $\mu(S)$. It takes the form of vertical line when $m=0$, then becomes deformed in hyperbolic branch towards the left by increasing the value $m$ (figure 3.2 ). The functions $H_{1}\left(S_{1}\right), H_{2}\left(S_{2}\right)$ and $G\left(S_{1}\right)$ do not depend on the kinetic $\mu(S)$. Their graphs do not change when $m$ is changed. These functions can be written in the form:

$$
\begin{gathered}
S=G\left(S_{1}\right)=\left(S_{1 i n}-S_{1}\right)\left(C_{1}^{\prime}+\frac{C_{2}^{\prime}}{\mu_{1}\left(S_{1}\right)}\right) . \\
S=H_{i}\left(S_{1}\right)=C_{1}+\left(S_{1 \text { in }}-S_{1}\right)\left(C_{2}+\frac{C_{3}}{\mu_{1}\left(S_{1}\right)}\right) .
\end{gathered}
$$

Where:

$$
\begin{gathered}
C_{1}^{\prime}=\frac{b_{3}+b_{1}}{k_{1} B}, \quad C_{2}^{\prime}=\frac{D_{0}-b_{1}\left(D_{0}+D_{1}\right)}{k_{1} B}, \\
C_{1}=\frac{A}{B}\left(S_{2 i n}-\lambda_{2}^{i}\right), \quad C_{2}=\frac{A_{1}-A_{2}}{k_{1} B} \\
C_{3}=\frac{A_{2}\left(D_{0}+D_{1}\right)+D_{0}}{k_{1} B}
\end{gathered}
$$

Before studying the graphical forms of the functions, we present the following inequalities which are imposed by the biological mass balance principle.

$$
\begin{aligned}
& k_{1} \geq 1+b_{3}+k_{2} \\
& k_{3} \geq 1+b_{4} \\
& b_{1} \geq 1+b_{2}
\end{aligned}
$$

This expresses the fact that the quantity of biomass and products produced is always smaller than the quantity of substrate consumed.

Since the SMP are slowly produced and degraded in the bioreactor, we can write,
- $k_{1}>b_{1}$ : The degraded quantity $S$ of SMP is smaller than the degraded quantity $S_{1}$ of substrate.

- $k_{2}>b_{2}$ : The produced quantity $S_{2}$ of VFA from $S_{1}$ is higher than the produced quantity from the SMP.

- $k_{2}>b_{3}$ : The produced quantity $S_{2}$ of VFA from $S_{1}$ is higher than the produced quantity $S$ of SMP from $S_{1}$.

- $b_{4}<k_{1}, k_{2}, k_{3}$ : The produced quantity $S$ of SMP from $S_{2}$ is small, the most part of $S_{2}$ is converted in biogas.

Because $k_{2}>b_{2}$, we have:

$$
C_{2}>0
$$

and from (13), we can deduce easily that the yield

$$
A<1 \text {, }
$$

which confirms a biological principle ( $A$ given by $(9)$ represents the quantity of SMP produced on the quantity of $S_{2}$ degraded). Now, from (14) and with the inequality $A<1$, we conclude that $b_{1}>A b_{2}+\frac{D_{0}}{D_{0}+D_{1}}$, which forces

$$
C_{3}<0 \text {. }
$$

Since $0<S_{1}<S_{1 i n}$ (no washout), and under the conditions $C_{1}^{\prime}>0, C_{2}^{\prime}<0, C_{2}>0, C_{3}<0$ and $C_{3}>-m_{1} C_{2}$, there exists sub-domains of $\left.S_{1} \in\right] 0, S_{1 i n}$, such that $S=G\left(S_{1}\right)$ and $S=H_{i}\left(S_{1}\right)$ are positive and are limited (we have a limited production of $S$ ).

The graphs of the functions $G, H_{1}$ and $H_{2}$ are as it is shown in figure 3.

We note on all the following figures:

$\lambda_{1}=\mu^{-1}\left(D_{0}+D_{1}\right)$.

$S_{1}^{H_{1}=0}$ : the greatest value of $S_{1}$ such that, $H_{1}\left(S_{1}^{H_{1}=0}\right)=0$.

$S_{1}^{H_{2}=0}$ : the greatest value of $S_{1}$ such that, $H_{2}\left(S_{1}^{H_{2}=0}\right)=0$.

We remark from the formula $(7)$ that $G\left(S_{1 i n}\right)=0$.

$S_{1 \text { in }}$ can take three positions compared to $S_{1}^{H_{1}=0}$ and $S_{1}^{H_{2}=0}$.

- Case 1: $S_{1 i n} \leq S_{1}^{H_{2}=0}<S_{1}^{H_{1}=0}$.

- Case 2: $S_{1}^{H_{2}=0}<S_{1 i n} \leq S_{1}^{H_{1}=0}$.

- Case 3: $S_{1}^{H_{2}=0}<S_{1}^{H_{1}=0}<S_{1 i n}$.

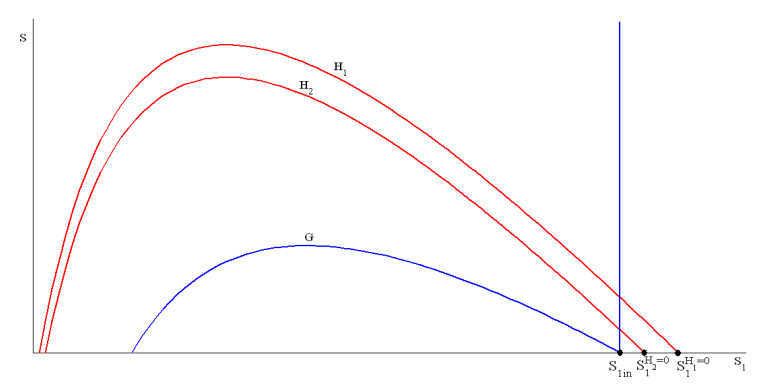

Fig. 3. The position of $S_{1 \text { in }}$ compared to $S_{1}^{H_{2}=0}$ and $S_{1}^{H_{1}=0}$

For illustration, we present on the figure 3 the case 1 .

We will analyse in the following the case 2 , the other cases can be analyzed in the same way (this case is given as example according to the selected set of parameters of table 1).

We trace all the graphs $F, G$ and $H_{i}$ together and we look 
at the possible positions of $\lambda_{1}$ compared to $S_{1 i n}, S_{1}^{H_{1}=0}$ and $S_{1}^{H_{2}=0}$, by changing only one parameter of the model, the other parameters being fixed. We change $K_{1}$ and we consider three sub-cases as it is shown by the figures 3.2 , 3.2 and 3.2 (other cases can exist according to the selected biological parameters)

- Case 2.1: $\lambda_{1}<S_{1}^{H_{2}=0}<S_{1 \text { in }}<S_{1}^{H_{1}=0}$.

- Case 2.2: $S_{1}^{H_{2}=0}<\lambda_{1}<S_{1 \text { in }}<S_{1}^{H_{1}=0}$.

- Case 2.3: $S_{1}^{H_{2}=0}<S_{1 i n}<S_{1}^{H_{1}=0}<\lambda_{1}$.

The intersections of the graphs $F$ with $G$ and $H_{i}$ give the solution $\left(S_{1}, S\right)$ of the equations systems (6-7) and (6-8) and thereafter, allow to calculate the equilibria according to the formulas of lemmas 10 and 11.

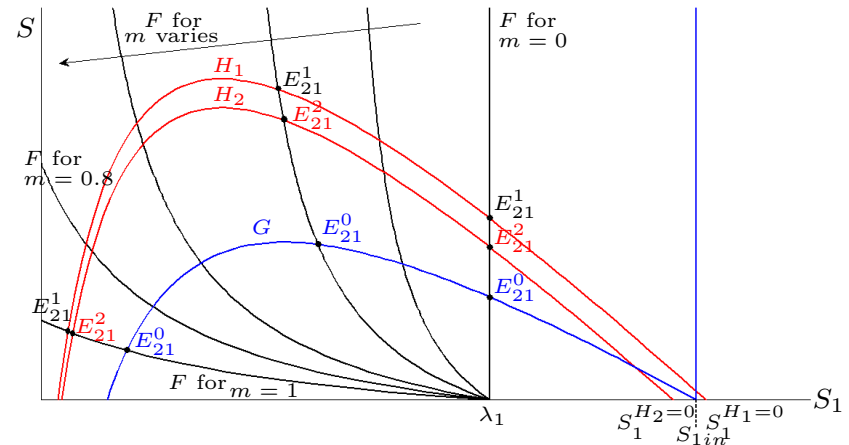

Fig. 4. The intersections of $F$ with $G, H_{i}$ in the case 2.1

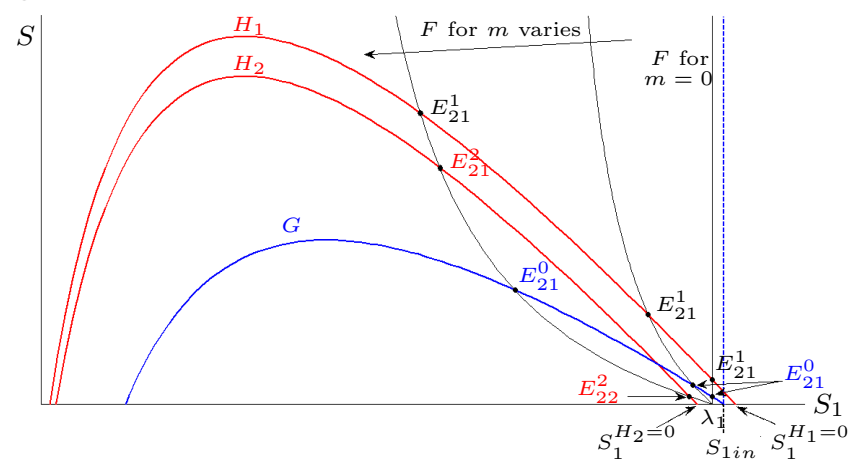

Fig. 5. The intersections of $F$ with $G, H_{i}$ in the case 2.2

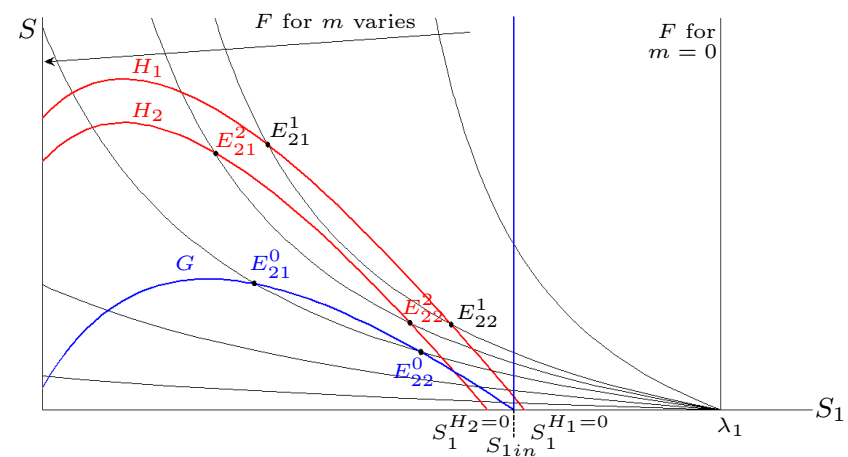

Fig. 6. The intersections of $F$ with $G, H_{i}$ in the case 2.3

\subsection{Simulation results and discussions}

To illustrate our approach, we perform simulations with nominal parameter values given by the table 1 .

The parameters of the model part without SMP (initial model AM2) have values equal or close to the AMOCO values (Bernard et al. (2001)). The other parameters of the part modeling the SMP, have values satisfying the below inequalities between $k_{i}$ and $b_{i}$ and, the inequalities (12), (13) and (14) (by transforming the coefficients units in $g / g)$.

Table 1. Nominal parameters values

\begin{tabular}{cccc} 
Parameters & Nominal values & Parameters & Nominal values \\
\hline$m_{1}$ & 1.2 & $k_{3}$ & 268 \\
$K_{1}$ & change & $b_{1}$ & 5 \\
$m_{2}$ & 1.5 & $b_{2}$ & 10 \\
$K_{2}$ & 5 & $b_{3}$ & 7 \\
$K_{I}$ & 15 & $b_{4}$ & 5 \\
$\beta$ & 0.6 & $m$ & {$[0 . .1]$} \\
$k_{1}$ & 25 & $K$ & 3 \\
$k_{2}$ & 250 & & \\
\hline
\end{tabular}

The values of the operating parameters are.

$$
D=1, D_{0}=0.25, D_{1}=0.25, S_{1 \text { in }}=10, S_{2 i n}=10
$$

We are interested in this paper in equilibria of the case shown by the figure 3.2 in which we have three equilibria. For $m=0$, the function $F$ is a vertical line on the left of $S_{1 i n}$ and intersects the functions $G$ and $H_{i}$ in three points, which gives the same solution $\lambda_{1}$ on the $x$-axis and three corresponding solutions $S^{*}$ on the $y$-axis. In fact, for $m=0$, we have exactly the case of the AM2 model with three equilibria in the phase plans $\left(S_{1}, X_{1}\right)$ and $\left(S_{2}, X_{2}\right)$ as it is shown by the figure 7 on the left. In this case, $X_{1}$ has the same values.
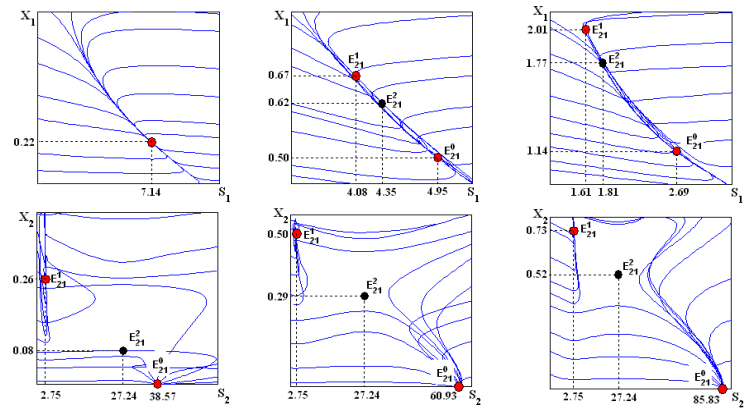

Fig. 7. The phase plans $\left(S_{1}, X_{1}\right)$ and $\left(S_{2}, X_{2}\right)$ for $m=0$ (left), $m=0.27$ (center) and $m=0.8$ (right)

For increasing values of $m$, the $X_{1}$ concentration increases because the $S_{1}$ concentration decreases. In the cases of equilibria $E_{21}^{1}$ and $E_{21}^{2}$, the $X_{2}$ concentration increases if $m$ increases and in the case of the washout equilibrium $E_{21}^{0}$, it is $S_{2}$ concentration which increases with $m$ (figure 7 on the center and on the right). The biomasses growth on the SMP can modify the system equilibria.

To study the stabilities of these equilibria, we calculated the Jacobian of system $f$ given by (1-5) and we replaced equilibria by their numerical values calculated from the graphs intersections. We calculated the eigenvalues for each equilibrium. Equilibria and stability are given by the following theorem.

Theorem 12. . In the case 2.1, the system (1-5) can have three equilibria:

- $E_{21}^{1}=\left(\lambda_{21}^{1}, X_{1,21}^{1}, \lambda_{2}^{1}, X_{2,21}^{1}, S_{21}^{1}\right)$ : Stable 
- $E_{21}^{2}=\left(\lambda_{21}^{2}, X_{1,21}^{2}, \lambda_{2}^{2}, X_{2,21}^{2}, S_{21}^{2}\right):$ Unstable

- $E_{21}^{0}=\left(\lambda_{21}^{0}, X_{1,21}^{0}, S_{2,21}^{0}, 0, S_{21}^{0}\right)$ : Stable

Where:

$\left(\lambda_{21}^{0}, S_{21}^{0}\right)$ and $\left(\lambda_{21}^{1}, S_{21}^{1}\right),\left(\lambda_{21}^{2}, S_{21}^{2}\right)$ are the solutions of equations systems (10) and (11) respectively.

$\left(X_{1,21}^{0}, X_{1,21}^{1}, X_{1,21}^{2}\right)$, and $\left(X_{2}=0, X_{2,21}^{1}, X_{2,21}^{2}\right)$ are the values of $X_{1}$ and $X_{2}$ given by the lemma 10 and 11. $\left(S_{2,21}^{0}\right)$ is the quantity of $S_{2}$ given by the lemma 10 .

We can also note in the figure 7 that equilibria $E_{21}^{1}$ and $E_{21}^{0}$ are attractive and locally stable, while equilibrium $E_{21}^{2}$ is unstable.

According to these results, we conclude that the original system AM2 perturbed by the introduction of SMP keeps the number and the nature of its equilibria. Only their values change with $m$. For values of $m$ close to 1 (sufficiently larger than 0 ), we have high concentrations of $X_{1}$ and $X_{2}$, lower concentrations of $S_{1}, S_{2}$ and $S$, which is important in this case. But, let us note in the figure 3.2 that for such values of $m$, the equilibria $E_{21}^{1}$ and $E_{21}^{2}$ approach and thus the attraction basin of the operational equilibrium is restricted, which weakens system stability. It is important from practical point of view to synthesize control laws stabilizing the system around the operational positive equilibrium $E_{21}^{1}$.

We remind that according to the biological parameters used, we can have other cases of the positions of the numbers $\lambda_{1}, S_{1 i n}, S_{1}^{H_{2}=0}$ and $S_{1}^{H_{1}=0}$ and thus the number and the nature of equilibria changes according to each case. For example, in the case of the figure 3.2 , we can have nine equilibria of which four are stable, the system has a quadri-stability.

\subsection{SMP influence on the equilibrium $\lambda_{1}$ of acidogenesis}

We noted that in absence of the growth on the SMP, i.e. when $m=0$, the AM2b model behaves exactly as the AM2 model. If the input organic matter $S_{1 i n}$ changes, then the value of equilibrium $\lambda_{1}$ remains unchangeable as it is shown by the figure 3.4 (we present only the graphs of $F$ and $H_{1}$ ).

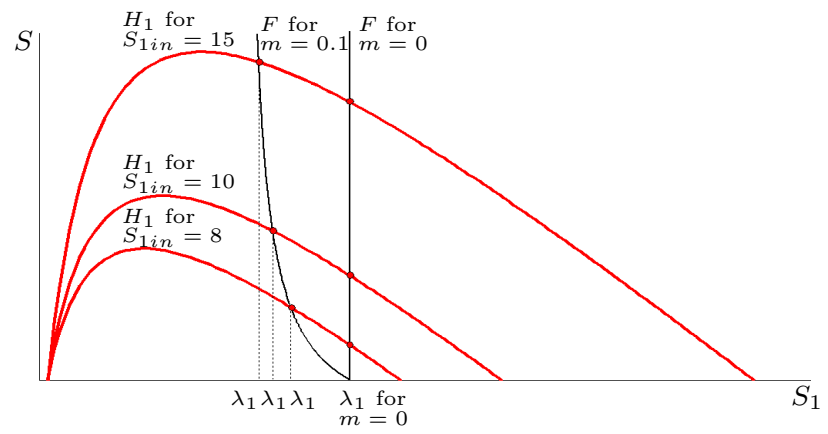

Fig. 8. Equilibrium $\lambda_{1}$ according to $S_{1 \text { in }}$ for $m=0$ and $m=0.1$

For the case $m \neq 0$, we remark that if $S_{1 \text { in }}$ increases, then the value of $\lambda_{1}$ decreases. This shows that the SMP integration in the model, can predict the change of equilibrium $\lambda_{1}$, which can occur, when the influent concentration $S_{1 \text { in }}$ of the organic matter changes. In fact, the equilibria $\lambda_{1}$ of the AM2b model are calculated by the resolution of (10) and (11), which contain explicitly the parameter $m$ in the function $F(S)$. On the contrary in the
AM2 model, there is no information on the SMP in the expression of equilibrium $\lambda_{1}$.

\section{CONCLUSION}

This paper presents the development and the analysis of a mathematical model for an AMBR. The model is based on the modification of the AM2 model of anaerobic digestion proposed in Bernard et al. (2001) and analyzed in Benyahia et al. (2010). Under general assumptions, it is shown that the modified AM2 model can keep the number and the nature of its equilibria. However, under certain operating conditions or if biological parameters values other than the default ones are used, the nature of the AM2b equilibria changes. Perspectives of the work include the characterization of the qualitative properties of the new proposed model, notably when the model exhibits multi-stability property.

\section{REFERENCES}

A.N.L. Ng, A.S. Kim. A mini-review of modeling studies on membrane bioreactor (MBR) treatment for municipal wastewaters. Desalination, 212: 261-281, 2007.

B. Benyahia, T. Sari, B. Cherki, J. Harmand. Equilibria of an anareobic wastewater treatment process and their stability. CAB'10, Leuven, 7-9 Juillet 2010.

C.S. Laspidou, B.E. Rittmann. A unified theory for extracellular polymeric substances, soluble microbial products, and active and inert biomass. Water Research, 36: 2711-2720, 2002.

D.R. Noguera, N. Araki, B.E. Rittmann. Soluble Microbial Products (SMP) in Anaerobic Chemostats. Biotechnol. Bioeng., 44: 1040, 1994.

D.J. Barker, D.C. Stuckey. A review of soluble microbial products (SMP) in wastewater treatment systems. $W a-$ ter Ressources, 33 (14): 3082-3082, 1999.

D.J. Barker, D.C. Stuckey. Modelling of soluble Microbial Products in Anaerobic Digestion: the effect of Feed Strength and Composition. Water Environ. Res., 73 (2): 173-184, 2001.

G. Lyberatos, I.V. Skiadas. Modelling of anaerobic digestion - A review. RGlobal Nest: the Int. J, 1, (2): 63-76, 1999.

O. Bernard, Z. Hadj-Sadock, D. Dochain, A. Genovesi, J.P. Steyer. Dynamical Model Development and Parameter Identification for an Anaerobic Wastewater Treatment Process. Biotechnology and Bioengineering, 75: 424-438, 2001.

P. Le-Clech, V. Chen, T.A.G. Fane. Fouling in membrane bioreactors used in wastewater treatment, Journal of Membrane Science. Journal of Membrane Science, 284: $17-53,2006$.

S.F. Aquino, D.C. Stuckey. Integrated model of the production of soluble microbial products (SMP) and extracellular polymeric substances (EPS) in anaerobic chemostats during transient conditions. Biochemical Engineering Journal, 38: 138-146, 2008. 